avances

en ciencias e

ingenierías

II Simposio
Latinoamericano o de Aplicaciones Nucleares

en la

Agricultura

\title{
Efecto de la irradiación gamma sobre las características de calidad y la durabilidad de la papa (Solanum tuberosum) almacenada
}

\author{
Elena Villacrés ${ }^{1 *}$, Mishel Yanez ${ }^{1,2}$, María Belén Quelal', Trosky Yánez² \\ ${ }^{1}$ Instituto Nacional de Investigaciones Agropecuarias (INIAP). Estación Experimental Santa Catalina, \\ Departamento de Nutrición y Calidad de Alimentos, Mejía, Ecuador. \\ ¿Universidad Central del Ecuador (UCE). Carrera de Química de Alimentos. Quito, Ecuador. \\ *Autor para correspondencia/Corresponding autor, e-mail: elenavillacres9@hotmail.com
}

\section{Effect of gamma irradiation on the quality characteristics and durability of stored potato (Solanum tuberosum)}

\begin{abstract}
Resumen
A través del monitoreo y evaluación de las características físico-químicas, se estimó la durabilidad de tres variedades de papa (chola, chaucha y ratona), las cuales recibieron un tratamiento de irradiación (120 Gy, 34.68 min) y se almacenaron por 40 días, bajo condición de ambiente $\left(12^{\circ} \mathrm{C}, 70 \%\right.$ humedad relativa) y refrigeración $\left(7^{\circ} \mathrm{C}, 70 \%\right.$ humedad relativa). Los resultados se compararon con los de los tubérculos que no fueron irradiados y se almacenaron bajo similares condiciones. En general, durante el almacenamiento, la intensidad respiratoria y varios nutrientes disminuyeron, mientras que la materia seca, los azucares reductores y el tamaño de los brotes se incrementaron. Sin embargo, estos cambios fueron menos drásticos en la variedad chola irradiada y almacenada en refrigeración, que presentó mayor contenido de humedad, menor pérdida de nutrientes, baja intensidad respiratoria y ausencia de brotes a los 40 días de monitoreo y prueba, proyectando su durabilidad hasta 106 días, bajo las condiciones mencionadas. Un comportamiento diferente presentó la variedad chaucha amarilla, sin tratamiento de irradiación y almacenada en condiciones de ambiente $\left(12^{\circ} \mathrm{C}\right.$ y $70 \%$ de humedad relativa), que presentó crecimiento de brotes a partir del quinto de almacenamiento con mayores pérdidas de nutrientes y degradación de las características físicas. Se concluyó que la irradiación y refrigeración combinadas, ayudarían a extender la durabilidad de la papa hasta 44 días (ratona), 114 días (chaucha) y 106 días (chola).
\end{abstract}

Editado por /

Edited by:

Gabriela Albán

Recibido /

Received:

$10 / 08 / 2020$

Aceptado /

Accepted:

$31 / 06 / 2020$

Publicado en línea /

Published online:

01/04/2021
Palabras clave: Brotes, Dormancia, Intensidad respiratoria, Nutrientes, Tegumento.

\section{Abstract}

Through the monitoring and evaluation of the physical-chemical characteristics, the durability of three potato varieties (chola, yellow chaucha and ratona) was estimated, which received an irradiation treatment (120 Gy, $34.68 \mathrm{~min}$ ) and were stored for 40 days, under ambient conditions $\left(12^{\circ} \mathrm{C}, 70 \%\right.$ relative humidity) and refrigeration $\left(7^{\circ} \mathrm{C}, 70 \%\right.$ humidity). The results were compared with those of the tubers that were not irradiated 
and were stored under similar conditions. In general, during storage, respiratory intensity and various nutrients decreased, while dry matter, reducing sugars, and shoot size increased. However, these changes were less drastic in irradiated and refrigerated Chola variety, which presented higher moisture content, less loss of nutrients, low respiratory intensity and absence of sprouts after 40 days of monitoring and testing, projecting its durability up to 106 days, under the conditions mentioned. A different behavior showed the yellow chaucha variety, without irradiation treatment and stored under ambient conditions $\left(12^{\circ} \mathrm{C}\right.$ and $70 \%$ relative humidity), which had sprouts growth from the fifth of storage, greater losses of nutrients and degradation of the characteristics physical. It was concluded that the application of irradiation and refrigeration combined, helped to extend the durability of the potato up to 44 days (ratona), 114 days (chaucha) and 106 days (chola).

Keywords: Dormancy, Nutrients, Respiratory intensity, Sprouts, Tegument.

\section{INTRODUCCIÓN}

La papa es el tercer cultivo alimenticio más importante a nivel mundial y ha sido recomendada por la Organización de las Naciones Unidas para la Agricultura y la Alimentación como un rubro estratégico de seguridad alimentaria para enfrentar el crecimiento exponencial de la población y los problemas de suministro de alimentos. En el 2016, Ecuador presentó una producción de 421000 t, de este volumen, aproximadamente el $81 \%$ es comercializado para consumo en fresco y el resto es utilizado por la industria alimenticia para el procesamiento de papa frita en forma de hojuelas o bastones [2]. La papa contribuye a la generación de ingresos de 82000 familias productoras de un total de 90 cantones de la Sierra ecuatoriana [1]. En su composición química destaca el alto contenido de humedad (70-80\%) por lo que es muy perecible y se dificulta su transporte. En la postcossecha las pérdidas anuales de los tubérculos varían entre 10-15\% y en algunas variedades la pérdida de la dormancia que se manifiesta con el aparecimiento de brotes, es uno de los factores más limitantes [5]. Los factores que contribuyen al deterioro cualitativo y cuantitativo de la papa podrían agruparse en cuatro categorías que incluyen físico, fisiológicos, microbiológicos y entomológicos [3]. Las tasas de respiración, transpiración y la presencia de microrganismos contribuyen a los procesos fisiológicos que aceleran el deterioro [4]. El manejo postcosecha de las variedades susceptibles a la brotación es muy importante, ya que puede ayudar a retrasar este proceso y aumentar la durabilidad para el consumo así como esperar una mejora en el precio de venta, lo cual es vital para la economía de los agricultores dedicados a este rubro. Los métodos de almacenamiento ayudan a prolongar el período de dormancia y retardar o inhibir cambios químicos indeseables. El almacenamiento refrigerado es limitado por el alto costo. Las bajas temperaturas de almacenamiento también inducen el endulzamiento de la papa, debido al incremento de azúcares totales y reductores, lo que limita su uso para la elaboración de chips [3]. Los métodos químicos se basan en el uso de productos para retrasar la brotación de los 
tubérculos [6], con este fin se utiliza el ácido abscísico (ABA) y el etileno. La inhibición del crecimiento de brotes (ICB), depende de factores como, la variedad, condiciones ambientales de almacenamiento, los estados fisiológicos de la planta cosechada, presencia de compuestos fenólicos, metabolismo de azucares, la cantidad y número de aplicaciones [7]. La irradiación gamma es un método conocido para eliminar o inactivar las causas del deterioro sin efectos adversos sobre la nutrición y calidad sensorial de los alimentos [3]. Esta técnica puede extender la vida útil, reducir el requisito de productos químicos para la conservación y control de plagas, esterilizar productos que luego se pueden almacenar sin refrigeración, retrasar la maduración de frutas y hortalizas, limitar el deterioro de la calidad de cultivos almacenados como tubérculos y bulbos, evitando la brotación postcosecha [8]. El problema crítico, es encontrar el óptimo nivel de radiación que puede cumplir con el requisito de conservación sin causar graves alteraciones químicas en los alimentos, que afecten su aceptabilidad física, organoléptica y su estado sanitario. Estudios realizados en varios países, indican que dosis entre 50-150 Gy aplicados durante el periodo de dormancia son recomendados para el control de brotes en los tubérculos [9]. La duración del periodo de dormancia de la papa es ligeramente afectado por la temperatura de almacenamiento pero es significativamente dependiente de la variedad [10]. Generalmente las fuentes de radiación son los rayos del núcleo excitado de elementos como el Cobalto 60 y el Cesio 137, siendo el primero el más utilizado en aplicaciones industriales [11]. Los rayos gamma son muy penetrantes y en la mayoría de los alimentos son eficaces a profundidades de incluso $20 \mathrm{~cm}$. La Organización Mundial de la Salud (OMS) ha autorizado dosis de radiación de incluso 7 KGy por ser absolutamente inocuas para el consumo humano [11]. La aceptación pública del concepto de la irradiación de alimentos no ha tenido acogida en algunos países. El miedo a la guerra termonuclear y ciertos accidentes como el sobrevenido en "Three Mile Island" en los Estados Unidos de América y en Chernobyl en la URSS han hecho que mucha gente desconfíe del uso de la energía nuclear con diversos propósitos, aunque se trate de algo tan conveniente como mejorar la cantidad y la calidad de los alimentos. Esa desconfianza, se basa a menudo en la falta de información y en la confusión entre el proceso de irradiación y la contaminación radiactiva. En algunas partes del mundo, donde la irradiación de alimentos lleva practicándose desde hace muchos años, ni el público ni los que influyen en la opinión pública no están bien informados sobre el proceso [23], por lo que, el objetivo de este estudio fue evaluar el efecto de la irradiación gamma y dos condiciones de almacenamiento sobre algunas características físico-químicas y la durabilidad de tres variedades de papa. Información que puede contribuir a valorar los efectos de esta tecnología en la conservación de la calidad y mejora de la inocuidad no solo de la papa sino también de otros alimentos perecibles.

\section{MATERIALES Y MÉTODOS}

Para este estudio, se utilizaron variedades de papa de consumo habitual en la Sierra ecuatoriana (chola) y otras que históricamente presentan un corto periodo de dormancia (chaucha amarilla y ratona). Estas variedades se cultivaron en la provincia de Cotopaxi, parroquia Toacazo en la comunidad Wintza. La irradiación de los tubérculos se realizó en la Planta de la Subsecretaria de control y aplicaciones nucleares (SCAN), adscrita al Ministerio de Electricidad y Energías Renovables (MEER), ubicada en Aloag, Pichincha. 
El almacenamiento, monitoreo y los análisis físico-químicos se realizaron en el Departamento de Nutrición y Calidad de la Estación Experimental Santa Catalina. Una vez cosechados los tubérculos fueron transportados a la Estación Santa Catalina, donde se clasificaron, seleccionaron y lavaron. Se trabajó con tubérculos de peso medio entre 41-60 g, que se lavaron con agua destilada y se escurrieron con la ayuda de papel toalla. Luego de estas operaciones, se determinaron las características físico-químicas de los tubérculos al tiempo cero posteriormente la muestra global fue divida en dos partes, una para el tratamiento de irradiación (120 Gy, 34.68 min) y otra sin irradiación. Tomando como referencia los resultados del estudio realizado por Ruíz-Ramos [12], se estableció la dosis y el tiempo de aplicación de la radiación gamma, a través de una fuente de Cobalto 60. Los lotes de tubérculos experimentales se almacenaron separadamente en una cámara bajo condiciones ambientales $\left(12^{\circ} \mathrm{C}, 70 \%\right.$ de humedad relativa) y en refrigeración $\left(7^{\circ} \mathrm{C}, 70 \% \mathrm{HR}\right)$. Se realizaron muestreos cada cinco días por un periodo de 40 días y se analizaron las siguientes características físico-químicas:

\section{Materia Seca}

La materia seca se determinó con base a la pérdida de peso que experimentó la papa sometida a desecación en estufa (Memmert, Schwabach, Germany) a una temperatura de $100-105^{\circ} \mathrm{C}$, hasta peso constante o durante 24 horas. La materia seca resultó de sustraer al peso total de la muestra el contenido en humedad, según el método 930.15 A.O.A.C. [13].

\section{Color del tegumento}

La medición del color se realizó usando un espectrofotómetro portátil (Lange SpectroColor d/8 $8^{\circ}$ modelo LZM 268, Chelmsford, UK) basado en el sistema de color CIEL*, a*, $b^{*}$. Se midieron los siguientes atributos de la sensación visual del color: $L^{*}$ (luminosidad), $\mathrm{C}^{*}$ (croma), and $\mathrm{H}^{*}$ (matiz). La diferencia de color entre la papa fresca (tiempo 0), tratada (con y sin irradiación gamma) y almacenada fue calculada aplicando la siguiente ecuación: $\Delta \mathrm{E}=\left(\Delta \mathrm{L}^{*} 2+\Delta \mathrm{C}^{*} 2+\Delta \mathrm{H}^{*} 2\right)^{0.5}[14]$.

\section{Azúcares reductores}

Los tubérculos fueron triturados en una licuadora Oster por $3 \mathrm{~min}$, con alcohol etílico al 80\%, luego se filtró el conjunto y se aforó a $25 \mathrm{~mL}$; se tomó $1 \mathrm{~mL}$ de filtrado y se trató con $6 \mathrm{~mL}$ de ácido pícrico, este reactivo reacciona con los azúcares reductores formando picramato, de color amarillo, cuya intensidad se determinó a $510 \mathrm{~nm}$ en un espectrofotómetro UV-Visible (Evolution 201, Thermo Scientific, Madrid, España). El porcentaje de azúcares reductores se calculó en referencia a una curva de calibración construida de la lectura de absorbancia de una serie de soluciones de glucosa preparadas en alcohol etílico [13].

\section{Ácido ascórbico}

La determinación se realizó usando el método espectrofotométrico [15], para lo cual se utilizó una solución de ácido oxálico al 0.4\% y acetona al 20\%, pH 1.1. El ácido ascórbico de la muestra reacciona con el 2,6 dicloroindofenol (2,6 DCIP) y lo reduce. Conforme el 
reactivo de color es reducido, su coloración azul característica disminuye hasta rojo o grosella. El cambio de coloración es registrado a $520 \mathrm{~nm}$ y la concentración de ácido ascórbico de la muestra se calculó usando una curva de calibración que emplea el L-ácido ascórbico como estándar.

\section{Tamaño de los brotes}

Se tomó una muestra de 10 tubérculos por cada tratamiento y repetición, lo que representa el 60\% de la muestra global; se midió la longitud de los brotes con un paquímetro digital Mitutoyo (Suzano, Brasil) [16].

\section{Intensidad respiratoria}

El método se basa en la captura del anhídrido carbónico mediante carbonatación del hidróxido de bario. Se determinó la diferencia de captura de anhídrido carbónico entre el blanco y la muestra, este resultado se relacionó con el peso de la muestra y el tiempo de respiración en la cámara [17].

\section{Durabilidad}

La durabilidad de la papa se estimó con base a la metodología sugerida por Alvarado [18], según la cual, la condición de pérdida de calidad en la cual la papa se considera inaceptable para el consumo en fresco es inferior al 65\% de humedad. Luego se determinó el orden de reacción que permita predecir el tiempo en que se alcanzaría la condición de inaceptable y se calculó el tiempo de vida útil o durabilidad.

\section{Análisis Estadístico}

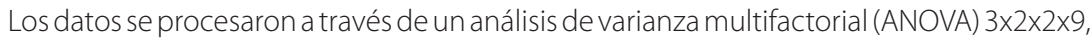
usando el programa estadístico INFOSTAT (Universidad de Córdova, Argentina), para la comparación de las medias con respecto a la variedad, condición de los tubérculos y ambiente de almacenamiento. La prueba de rango múltiple de Tukey fue usada para determinar diferencias significativas a un nivel del 5\%. Todos los análisis se hicieron por triplicado, los resultados están expresados como la media \pm desviación estándar. La estimación de la vida útil se realizó con base a las ecuaciones que relacionan la variación de la humedad con el tiempo de almacenamiento, Como límite crítico de aceptabilidad para el consumo de la papa en estado fresco, se estableció un 65\% de humedad [16].

\section{RESULTADOS Y DISCUSIÓN}

\section{Materia Seca}

La materia seca varió significativamente con la variedad de papa, el tratamiento, el tiempo y la condición de almacenamiento ( $p<0.05$ ). Tukey al $5 \%$ para la interacción "variedades $x$ tratamiento $x$ condición de almacenamiento x tiempo de almacenamiento" identificó 27 rangos de significación. En el primer rango con el menor contenido de materia seca se encuentra la variedad ratona no irradiada, almacenada al ambiente o refrigeración por un día con $17.06 \%$ y en el último rango de significación con el mayor 
contenido de materia seca se ubica la misma variedad no irradiada, almacenada al ambiente por 40 días, con 60.98\%. Este parámetro guarda relación directa con el contenido de humedad, el cual a su vez influye en la calidad y aceptabilidad de la papa para el consumo en estado fresco. Durante el almacenamiento los tubérculos perdieron humedad por deshidratación, debido a la diferencia entre la humedad relativa del aire y la del tubérculo, esta última corresponde a la del aire saturado (90\%). La tasa de evaporación fue mayor en los tubérculos no irradiados con respecto a los que recibieron radiación. La variedad ratona mostró un mayor contenido de materia seca almacenada bajo condiciones ambientales, (Figuras 1 y 2). Estos resultados evidencian el efecto de la irradiación en la disminución de la actividad metabólica de la papa y la velocidad de pérdida de humedad.

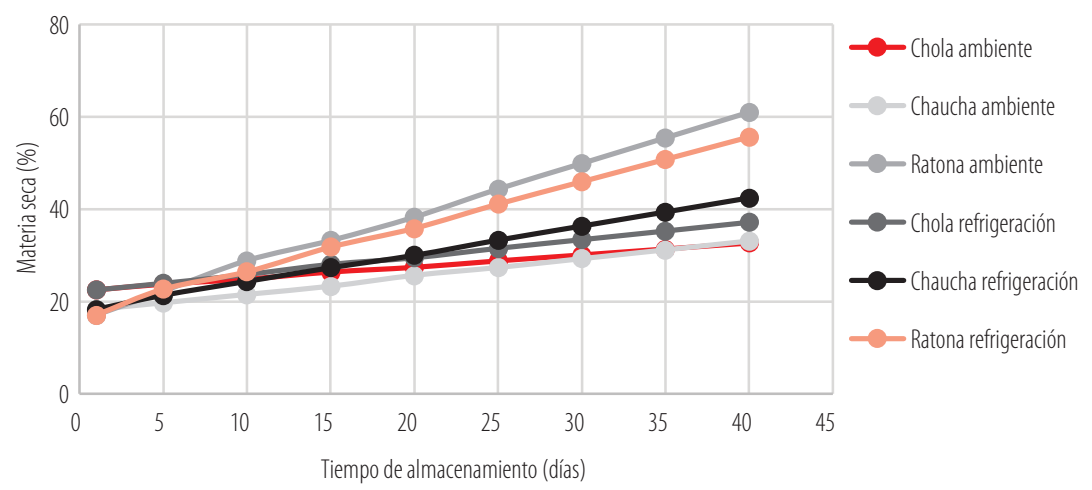

Figura 1. Variación de la materia seca con el tiempo de almacenamiento de la papa no irradiada

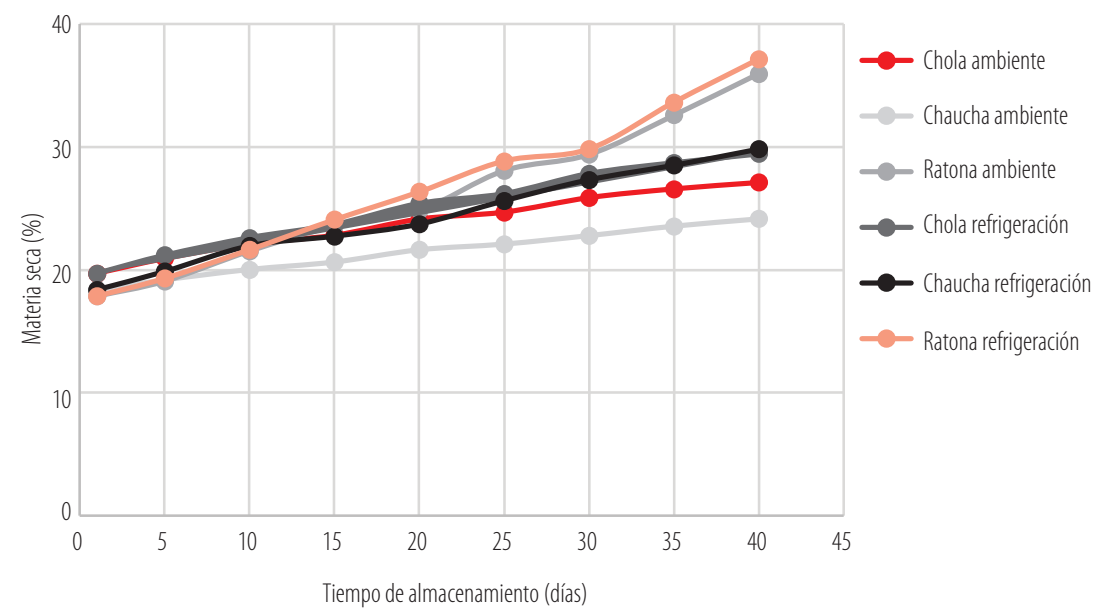

Figura 2. Variación de la materia seca con el tiempo de almacenamiento de la papa irradiada 


\section{Almidón y Azúcares reductores}

El almidón es el mayor componente de la materia seca y principal carbohidrato de reserva de la papa. Se sintetiza durante la formación del tubérculo y se degrada para proveer los carbohidratos necesarios para el crecimiento de los brotes [19]. En la papa almacenada ocurrieron cambios químicos importantes, entre los más relevantes se menciona la disminución del almidón y humedad, aumento del contenido azúcares y materia seca. Estos dos últimos variaron significativamente por efecto de la variedad, el tratamiento (con y sin irradiación), la condición (ambiente y refrigeración) y el tiempo de almacenamiento $(p<0.05)$ como se muestra en la Tabla 1. La prueba de Tukey al $5 \%$ para la interacción "variedades $x$ tratamiento $x$ condición de almacenamiento $x$ tiempo de almacenamiento" identificó 58 rangos de significación. En el primer rango con el mayor contenido de almidón se encuentra la variedad chaucha irradiada, almacenada al ambiente y en refrigeración por un día con 80.31\% y en el último rango de significación con el menor contenido de almidón se ubica la misma variedad no irradiada, almacenada en refrigeración por 40 días, con 43.23\%. En general, los tubérculos no irradiados, almacenados en refrigeración presentaron la mayor pérdida de almidón. Este fenómeno se revirtió con la irradiación y el almacenamiento al ambiente, bajo estas condiciones las variedades chola, ratona y chaucha presentaron pérdidas en el orden del 9.96, 14.34 y 17.34\%, respectivamente. Paralelo a la disminución de este nutriente, se registró un aumento de los azucares reductores y se alcanzó un promedio de $1 \mathrm{~g} / 100 \mathrm{~g}$ en la variedad chaucha no irradiada y almacenada en refrigeración. Al respecto, Rodríguez \& Moreno [6] indican que el almidón tiende a disminuir en los tubérculos almacenados a bajas temperaturas, debido a la hidrólisis de este compuesto en azucares o el inicio de la brotación. Tukey al $5 \%$ para la interacción "variedades $x$ tratamiento $x$ condición de almacenamiento $x$ tiempo de almacenamiento" identificó 50 rangos de significación. En el primer rango con el menor contenido de azúcares se encuentra la variedad chola irradiada y no irradiada, almacenada al ambiente y en refrigeración por 1 y 5 días con 7.62 mg/100 g y en el último rango de significación con el mayor contenido de azúcares reductores se ubica la variedad chaucha no irradiada, almacenada en refrigeración por 40 días, con 1.07 g/100 g. En general, la irradiación disminuyó la magnitud de cambio, efecto que fue notable en la variedad chola que presentó el menor contenido de azúcares reductores (44.48 mg/100 g) después de 40 días de almacenamiento al ambiente. Estos resultados concuerdan con las investigaciones realizadas por Claasens [19], quien encontró que durante la iniciación de la dormancia se produce una síntesis de almidón, mientras que al final de la misma predomina el desdoblamiento del compuesto hacia sus monómeros constituyentes, como la sacarosa. Viola et al. [20] asume que este cambio funcional permite la difusión de sacarosa en el apoplasto de tubérculos fuente, aumentando su disponibilidad para la absorción en el floema y el transporte hacia los brotes. 
Tabla 1. Variación del contenido de almidón y azucares reductores por efecto del tratamiento, condición y tiempo de almacenamiento de tres variedades de papa

\begin{tabular}{|c|c|c|c|c|c|c|c|c|}
\hline \multirow[b]{2}{*}{$\begin{array}{l}\text { Trata- } \\
\text { miento }\end{array}$} & \multirow[b]{2}{*}{$\begin{array}{c}\text { Almace- } \\
\text { namiento }\end{array}$} & \multirow[b]{2}{*}{ Días } & \multicolumn{3}{|c|}{ Almidón (\%) } & \multicolumn{3}{|c|}{ Azucares Reductores $(\mathrm{mg} / 100 \mathrm{~g})$} \\
\hline & & & Chola & Chaucha & Ratona & Chola & Chaucha & Ratona \\
\hline \multirow{18}{*}{ 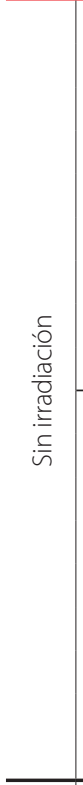 } & \multirow{9}{*}{ 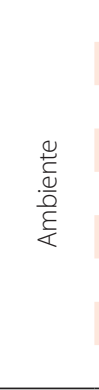 } & 1 & $76.37 \pm 0.16^{2}$ & $80.22 \pm 0.16^{\mathrm{a}}$ & $78.30 \pm 0.16^{x}$ & $8.41 \pm 1.48^{A}$ & $31.06 \pm 1.45^{A-F}$ & $16.46 \pm 1.88^{A-C}$ \\
\hline & & 5 & $74.17 \pm 0.16^{\mathrm{k}}$ & $76.83 \pm 0.16^{\circ}$ & $75.64 \pm 0.01^{2}$ & $19.21 \pm 0.36^{A-C}$ & $76.50 \pm 3.17$ & $64.78 \pm 2.26^{A-0}$ \\
\hline & & 10 & $72.06 \pm 0.10^{n 0}$ & $73.16 \pm 0.10^{\mathrm{m}}$ & $72.61 \pm 0.01^{100}$ & $41.04 \pm 1.05^{\mathrm{A-1}}$ & $180.92 \pm 2.37^{W-d}$ & $172.13 \pm 1.66^{1-b}$ \\
\hline & & 15 & $69.13 \pm 0.16$ & $70.87 \pm 0.16^{\mathrm{t}}$ & $70.05 \pm 0.16^{\text {ghi }}$ & $63.75 \pm 1.30^{4-0}$ & $251.15 \pm 3.00^{d^{d f}}$ & $275.16 \pm 1.14^{e-9}$ \\
\hline & & 20 & $66.1 \pm 0.16^{d-g}$ & $64.18 \pm 0.16^{\mathrm{PQ}}$ & $65.37 \pm 0.16^{N}$ & $85.68 \pm 2.84^{B-R}$ & $353.2 \pm 1.22^{i j}$ & $348.62 \pm 1.96^{9-i}$ \\
\hline & & 25 & $63.68 \pm 0.04^{0}$ & $61.31 \pm 0.03^{\cup}$ & $62.69 \pm 0.03^{2 R}$ & $105.19 \pm 1.17^{\curvearrowleft-U}$ & $431.68 \pm 1.58 \mathrm{j}-1$ & $445.67 \pm 1.48^{k-m}$ \\
\hline & & 30 & $61.00 \pm 0.02^{W W}$ & $57.35 \pm 0.01^{\mathrm{L}}$ & $59.41 \pm 0.02^{W}$ & $125.99 \pm 1.63^{\mathrm{M}-\mathrm{Y}}$ & $517.19 \pm 1.64^{m-0}$ & $536.97 \pm 1.83^{n-p}$ \\
\hline & & 35 & $58.34 \pm 0.02$ & $53.38 \pm 0.03^{b}$ & $56.13 \pm 0.02^{1 M}$ & $149.79 \pm 1.76^{R-a}$ & $602.7 \pm 1.46$ & $628.27 \pm 2.42^{0-2}$ \\
\hline & & 40 & $55.68 \pm 0.02^{\mathrm{MN}}$ & $49.42 \pm 0.04^{f}$ & $52.85 \pm 0.02^{D}$ & $168.59 \pm 3.03^{W-d}$ & $688.21 \pm 3.28^{5-4}$ & $719.57 \pm 1.91^{\text {tu }}$ \\
\hline & \multirow{9}{*}{ 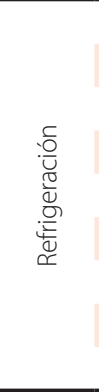 } & 1 & $76.37 \pm 0.16^{2}$ & $80.22 \pm 0.16^{a}$ & $78.30 \pm 0.16^{w}$ & $8.41 \pm 1.48^{\AA}$ & $31.06 \pm 1.45^{\text {A.F }}$ & $16.46 \pm 1.88^{A-C}$ \\
\hline & & 5 & $72.98 \pm 0.42^{m}$ & $78.20 \pm 0.16 y$ & $73.07 \pm 0.16^{\mathrm{m}}$ & $39.36 \pm 8.24^{A-1}$ & $132.88 \pm 2.63^{N-2}$ & $77.18 \pm 3.21^{\mathrm{aE}}$ \\
\hline & & 10 & $70.05 \pm 0.16 g^{\text {hi }}$ & $71.70 \pm 0.16^{\mathrm{pq}}$ & $69.13 \pm 0.16$ & $63.88 \pm 1.33^{A-0}$ & $236.14 \pm 1.12^{b-f}$ & $187.85 \pm 1.61^{Y-d}$ \\
\hline & & 15 & $68.03 \pm 0.16^{a}$ & $68.85 \pm 0.16$ & $66.84 \pm 0.01^{b c}$ & $94.15 \pm 1.21^{A-E}$ & $384.19 \pm 0.79^{-k}$ & $282.77 \pm 3,23^{f-h}$ \\
\hline & & 20 & $64.55 \pm 0.16^{P}$ & $61.70 \pm 0.16^{5}$ & $61.98 \pm 0.16^{R}$ & $128.77 \pm 2,28^{\mathrm{N}-\mathrm{Y}}$ & $549.12 \pm 3.10^{\mathrm{hi}}$ & $369.66 \pm 3.08$ \\
\hline & & 25 & $61.61 \pm 0.01^{\top}$ & $57.78 \pm 0.03^{K}$ & $57.94 \pm 0.02$ & $157.91 \pm 2.08^{R-a}$ & $664.35 \pm 1.49^{\circ p}$ & $468.27 \pm 2.37^{-n}$ \\
\hline & & 30 & $58.64 \pm 0.02^{x}$ & $52.93 \pm 0.05^{c}$ & $53.91 \pm 0.04^{A}$ & $188.65 \pm 2.24^{y-d}$ & $798.71 \pm 2.08^{p-r}$ & $563.36 \pm 1.82^{-n}$ \\
\hline & & 35 & $52.7 \pm 0.04^{\mathrm{E}}$ & $48.08 \pm 0.03^{F}$ & $49.88 \pm 0.02^{\mathrm{F}}$ & $219.39 \pm 1.98^{\mathrm{a}-\mathrm{f}}$ & $933.05 \pm 2.19^{w}$ & $658.46 \pm 2.96^{1-t}$ \\
\hline & & 40 & $52.7 \pm 0.04^{F}$ & $43.23 \pm 0.02^{H}$ & $45.86 \pm 0.02^{6}$ & $250.13 \pm 3.90^{c-f}$ & $1067.4 \pm 1.92^{x}$ & $753.55 \pm 1.23^{\text {wv }}$ \\
\hline \multirow{18}{*}{ 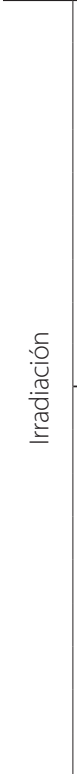 } & \multirow{9}{*}{ 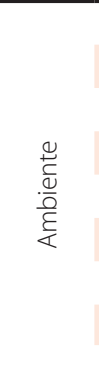 } & 1 & $76.37 \pm 0.161^{2}$ & $80.31 \pm 0.01^{A}$ & $79.21 \pm 0.01^{x}$ & $7.62 \pm 0.67^{\AA}$ & $18.34 \pm 2.62^{A-C}$ & $13.98 \pm 1.13^{\mathrm{AB}}$ \\
\hline & & 5 & $75.00 \pm 0.02^{4}$ & $78.29 \pm 0.02^{y}$ & $77.59 \pm 0.02^{2}$ & $9.42 \pm 1.48^{A}$ & $28.98 \pm 1.82^{\mathrm{AE}}$ & $19.99 \pm 1.51^{A-D}$ \\
\hline & & 10 & $73.62 \pm 0.16^{\mathrm{m}}$ & $75.73 \pm 0.16^{2}$ & $75.64 \pm 0.01^{3}$ & $12.91 \pm 1.32^{A B}$ & $47.29 \pm 2.23^{A-K}$ & $34.39 \pm 2.02^{A-G}$ \\
\hline & & 15 & $73.08 \pm 0.03^{\mathrm{m}}$ & $74.98 \pm 0.02^{4}$ & $74.76 \pm 0.02^{4}$ & $18.89 \pm 2.3^{3}$ & $69.09 \pm 2.59^{4-0}$ & $58.54 \pm 1.69^{A-N}$ \\
\hline & & 20 & $71.7 \pm 0.16^{o p}$ & $72.80 \pm 0.16^{n}$ & $73.16 \pm 0.01^{\mathrm{m}}$ & $22.25 \pm 0.74^{4-D}$ & $84.7 \pm 1.22^{B-R}$ & $68.63 \pm 1.90^{A-0}$ \\
\hline & & 25 & $71.16 \pm 0.02^{q 5}$ & $71.66 \pm 0.03^{q r}$ & $71.93 \pm 0.03^{\circ p}$ & $28.37 \pm 2.52^{A-E}$ & $109.2 \pm 1.63^{H \cdot x}$ & $97.1 \pm 1.55^{\mathrm{E}-\mathrm{T}}$ \\
\hline & & 30 & $69.95 \pm 0.16^{\mathrm{hi}}$ & $71.05 \pm 0.16^{15}$ & $70.60 \pm 0.16^{\text {tu }}$ & $31.82 \pm 1.61^{A-F}$ & $122.32 \pm 1.50^{-Y Y}$ & $112.65 \pm 1.91^{-x}$ \\
\hline & & 35 & $69.24 \pm 0.04$ & $68.35 \pm 0.02^{y z}$ & $69.10 \pm 0.03^{j}$ & $37.84 \pm 2.16^{\mathrm{A}-\mathrm{H}}$ & $149.3 \pm 1.91^{0-a}$ & $135.65 \pm 1.64^{0-z}$ \\
\hline & & 40 & $68.76 \pm 0.28^{x}$ & $66.38 \pm 0.16^{\text {cd }}$ & $67.85 \pm 0.16^{\mathrm{b}}$ & $44.48 \pm 1.25^{A-J}$ & $177.08 \pm 2.92^{2-d}$ & $163.3 \pm 1.40^{5-b}$ \\
\hline & \multirow{9}{*}{ 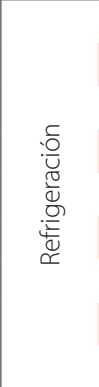 } & 1 & $76.37 \pm 0.160^{1}$ & $80.31 \pm 0.01^{A}$ & $79.21 \pm 0.01^{\mathrm{B}}$ & $7.62 \pm 2.62^{A}$ & $18.34 \pm 2.62^{A-C}$ & $13.98 \pm 1.13^{\mathrm{AB}}$ \\
\hline & & 5 & $74.57 \pm 0.02^{\mathrm{k}}$ & $78.36 \pm 0.02^{x}$ & $77.80 \pm 0.02^{y}$ & $15.36 \pm 2.97^{A B}$ & $43.63 \pm 1.48^{\mathrm{A-1}}$ & $26.18 \pm 1.55^{A-E}$ \\
\hline & & 10 & $72.61 \pm 0.10^{n}$ & $75.18 \pm 0.16^{3}$ & $75.00 \pm 0.16^{4}$ & $22.95 \pm 2.93^{A-E}$ & $73.06 \pm 1.39^{A-p}$ & $48.45 \pm 1.33^{\mathrm{A}-\mathrm{L}}$ \\
\hline & & 15 & $71.92 \pm 0.02^{\circ p}$ & $73.83 \pm 0.02$ & $73.97 \pm 0.02$ & $29.85 \pm 0.5^{A-E}$ & $90.14 \pm 1.63^{c-s}$ & $66.49 \pm 1.46^{A-0}$ \\
\hline & & 20 & $70.05 \pm 0.16^{\mathrm{ghi}}$ & $71.97 \pm 0.160^{p}$ & $73.44 \pm 0.01^{\mathrm{m}}$ & $39.30 \pm 3.29^{A-1}$ & $119.43 \pm 1.34^{K}-Y$ & $79.89 \pm 1.59^{A-0}$ \\
\hline & & 25 & $69.26 \pm 0.02$ & $69.30 \pm 0.02$ & $70.13 \pm 0.02^{f-h}$ & $44.33 \pm 2.03^{\mathrm{A}-\mathrm{J}}$ & $136.65 \pm 2.24^{-2-z}$ & $106.8 \pm 1.50^{6}-W$ \\
\hline & & 30 & $68.12 \pm 0.16^{a}$ & $68.40 \pm 0.16^{y z}$ & $68.58 \pm 0.16^{y}$ & $53.74 \pm 1.77^{A-M}$ & $155.38 \pm 1.47^{R-a}$ & $118.82 \pm 1.49^{-Y}$ \\
\hline & & 35 & $66.61 \pm 0.02^{b c}$ & $64.78 \pm 0.02^{0}$ & $66.29 \pm 0.03^{\text {ce }}$ & $58.82 \pm 1.24^{4-N}$ & $183.17 \pm 1.66^{x-d}$ & $147.11 \pm 1.22^{0-a}$ \\
\hline & & 40 & $65.55 \pm 0.16^{e-9}$ & $61.52 \pm 0.16^{\top}$ & $63.63 \pm 0.16^{6 R}$ & $63.30 \pm 2.37^{A-0}$ & $205.43 \pm 1.27^{-\mathrm{e}}$ & $176.13 \pm 2.77^{U-c}$ \\
\hline
\end{tabular}


Tabla 2. Variación del ácido ascórbico y la intensidad respiratoria por efecto del tratamiento, condición y tiempo de almacenamiento de tres variedades de papa

\begin{tabular}{|c|c|c|c|c|c|c|c|c|}
\hline \multirow[b]{2}{*}{$\begin{array}{l}\text { Trata- } \\
\text { miento }\end{array}$} & \multirow[b]{2}{*}{$\begin{array}{c}\text { Almace- } \\
\text { namiento }\end{array}$} & \multirow[b]{2}{*}{ Días } & \multicolumn{3}{|c|}{ Ácido ascórbico $(\mathrm{mg} / 100 \mathrm{~g})$} & \multicolumn{3}{|c|}{ Intensidad respiratoria ( $\mathrm{mg} \mathrm{CO}_{2} / \mathrm{kg}^{*} \mathrm{~h}$ ) } \\
\hline & & & Chola & Chaucha & Ratona & Chola & Chaucha & Ratona \\
\hline \multirow{18}{*}{ 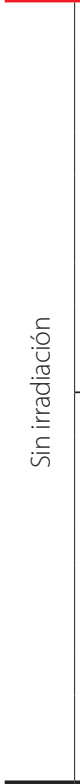 } & \multirow{9}{*}{ 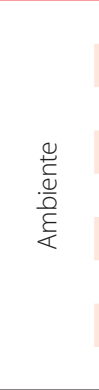 } & 1 & $7.31 \pm 0.08^{\mathrm{abc}}$ & $9.34 \pm 0.14^{a}$ & $7.93 \pm 0.24^{2-c}$ & $73.88 \pm 0.12^{\circ}$ & $83.28 \pm 0.54^{\mathrm{ab}}$ & $82.47 \pm 1.62^{d-0}$ \\
\hline & & 5 & $5.55 \pm 0.16^{m-x}$ & $5.40 \pm 0.06^{n-x}$ & $6.25 \pm 0.07^{\text {de }}$ & $65.23 \pm 1.10^{q-a}$ & $73.71 \pm 1.70^{y-m}$ & $70.69 \pm 0.45^{-0-0}$ \\
\hline & & 10 & $4.41 \pm 0.07^{\mathrm{m}-u}$ & $4.41 \pm 0.61^{1-u}$ & $5.03 \pm 0.03^{5-y}$ & $60.01 \pm 1.08^{s-h}$ & $64.52 \pm 0.87^{-c c}$ & $61.48 \pm 1.93^{s-f}$ \\
\hline & & 15 & $4.18 \pm 0.03^{-\mathrm{a}-1}$ & $3.98 \pm 0.33^{-0}$ & $3.58 \pm 0.05^{-\mathrm{r}}$ & $50.29 \pm 0.03^{\text {ce }}$ & $51.56 \pm 0.52^{b-d}$ & $52.01 \pm 0.48^{a-c}$ \\
\hline & & 20 & $3.52 \pm 0.03^{\mathrm{ab}}$ & $3.48 \pm 0.04^{a-f}$ & $3.61 \pm 0.13^{\mathrm{a}-\mathrm{q}}$ & $45.69 \pm 0.13^{d-9}$ & $44.08 \pm 0.63^{d-9}$ & $44.86 \pm 0.25^{e-h}$ \\
\hline & & 25 & $3.51 \pm 0.02^{a-c}$ & $3.47 \pm 0.02^{2-f}$ & $3.59 \pm 0.05^{2-9}$ & $37.11 \pm 0.01^{i-1}$ & $32.46 \pm 0.02-1$ & $33.49 \pm 0.01^{-1}$ \\
\hline & & 30 & $3.48 \pm 0.02^{\mathrm{a}-\mathrm{d}}$ & $3.44 \pm 0.04^{\text {a-e }}$ & $3.46 \pm 0.01^{\text {a-f }}$ & $29.70 \pm 0.01^{k-m}$ & $22.00 \pm 0.02^{1-n}$ & $23.76 \pm 0.02^{k-m}$ \\
\hline & & 35 & $3.46 \pm 0.01^{\text {af }}$ & $3.10 \pm 0.01^{\text {a-f }}$ & $3.42 \pm 0.02^{\text {ae }}$ & $22.30 \pm 0.02^{k-n}$ & $11.53 \pm 0.03^{1-p}$ & $14.03 \pm 0.02^{1-p}$ \\
\hline & & 40 & $3.42 \pm 0.02^{\mathrm{a}-\mathrm{e}}$ & $3.40 \pm 0.02^{a-d}$ & $3.39 \pm 0.01^{\mathrm{a}-\mathrm{f}}$ & $19.90 \pm 0.02^{1-0}$ & $1.07 \pm 0.01^{1-q}$ & $4.30 \pm 0.02^{1-q}$ \\
\hline & \multirow{9}{*}{ 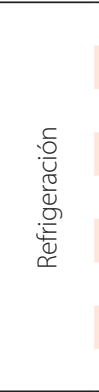 } & 1 & $7.31 \pm 0.08^{\mathrm{abc}}$ & $9.34 \pm 0.14^{d}$ & $7.93 \pm 0.24^{2-c}$ & $73.88 \pm 0.12^{-i-i}$ & $83.28 \pm 0.54^{\mathrm{ab}}$ & $82.47 \pm 1.62^{c-0}$ \\
\hline & & 5 & $5.38 \pm 0.10^{n-x}$ & $4.58 \pm 0.07^{h-t}$ & $4.73 \pm 0.38^{c-5}$ & $71,55 \pm 0.17^{2-n}$ & $76.84 \pm 0.05^{m-0}$ & $77.06 \pm 0.43^{1-0}$ \\
\hline & & 10 & $3.57 \pm 0.04^{a-5}$ & $3.56 \pm 0.02^{\mathrm{a}-5}$ & $3.76 \pm 0.02^{2-9}$ & $69.13 \pm 0.19^{\mathrm{a}-0}$ & $72.94 \pm 0.48^{y-m}$ & $71.54 \pm 0.19^{2-n}$ \\
\hline & & 15 & $3.52 \pm 0.03^{\mathrm{ab}}$ & $3.48 \pm 0.04^{a-d}$ & $3.61 \pm 0.13^{\mathrm{a}-q}$ & $66.86 \pm 0.19^{0-w}$ & $67.51 \pm 0.26^{n-5}$ & $66.96 \pm 0.25^{n-u}$ \\
\hline & & 20 & $3.50 \pm 0.02^{\mathrm{a}-\mathrm{f}}$ & $3.80 \pm 0.17^{a-p}$ & $3.47 \pm 0.03^{\mathrm{a}-\mathrm{e}}$ & $64.52 \pm 0.18^{r-c}$ & $63.51 \pm 0.22^{f-i}$ & $63.71 \pm 0.29^{r-c}$ \\
\hline & & 25 & $3.48 \pm 0.02^{a-f}$ & $3.73 \pm 0.05^{\mathrm{a}-q}$ & $3.44 \pm 0.02^{\text {a-e }}$ & $61.98 \pm 0.01^{-f f}$ & $57.82 \pm 0.03^{\text {sh }}$ & $57.73 \pm 0.03^{\text {sh }}$ \\
\hline & & 30 & $3.44 \pm 0.03^{\text {a-e }}$ & $3.62 \pm 0.07^{\mathrm{a}-q}$ & $3.41 \pm 0.01^{\text {a-f }}$ & $59.55 \pm 0.01^{1-0}$ & $52.75 \pm 0.01^{\mathrm{bb}}$ & $52.79 \pm 0.03^{\mathrm{a}}$ \\
\hline & & 35 & $3.40 \pm 0.01^{a-d}$ & $3.58 \pm 0.03^{\mathrm{a}-5}$ & $3.38 \pm 0.02^{\mathrm{a}-9}$ & $57.11 \pm 0.01^{1-0}$ & $47.69 \pm 0.02^{\mathrm{d}-f}$ & $47.85 \pm 0.02^{c-f}$ \\
\hline & & 40 & $3.36 \pm 0.02^{\mathrm{a}-9}$ & $3.47 \pm 0.03^{\text {ae }}$ & $3.37 \pm 0.03^{\mathrm{a}-9}$ & $54.68 \pm 0.01^{1-0}$ & $42.62 \pm 0.02^{h-k}$ & $42.91 \pm 0.02^{9-j}$ \\
\hline \multirow{18}{*}{ 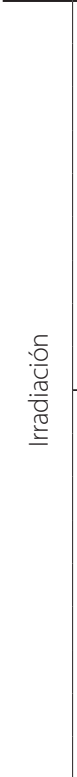 } & \multirow{9}{*}{ 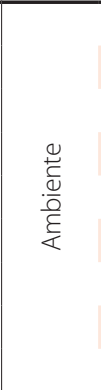 } & 1 & $7.23 \pm 0.08^{b-c}$ & $9.02 \pm 0.66^{y-a}$ & $8.29 \pm 1.47^{y-b}$ & $72.93 \pm 0.45^{y-m}$ & $83.38 \pm 0.28^{\mathrm{a}}$ & $82.46 \pm 2.12^{e-0}$ \\
\hline & & 5 & $6.15 \pm 0.06^{\mathrm{ef}}$ & $7.14 \pm 0.62^{\text {cd }}$ & $7.16 \pm 0.79^{c d}$ & $72.25 \pm 0.01^{y-m}$ & $80.77 \pm 0.02^{h-0}$ & $81.27 \pm 0.02^{9-0}$ \\
\hline & & 10 & $4.87 \pm 0.03^{c-5}$ & $5.30 \pm 0.86^{0-y}$ & $5.94 \pm 0.17^{f}$ & $71.41 \pm 0.48^{8^{-0}}$ & $77.40 \pm 0.28^{k-0}$ & $80.34 \pm 0.36^{i-0}$ \\
\hline & & 15 & $4.68 \pm 0.06^{\text {e-s }}$ & $4.99 \pm 0.20^{t-y}$ & $5.54 \pm 0.23^{m-x}$ & $69.73 \pm 0.02^{2-0}$ & $76.11 \pm 0.02^{\text {no }}$ & $76.83 \pm 0.02^{\text {no }}$ \\
\hline & & 20 & $4.49 \pm 0.06^{i-u}$ & $4.65 \pm 0.49^{f-5}$ & $5.22 \pm 0.22^{q-y}$ & $68.41 \pm 0.01^{b-0}$ & $74.33 \pm 0.64^{\circ}$ & $73.77 \pm 0.58^{w-j}$ \\
\hline & & 25 & $4.45 \pm 0.04^{k-u}$ & $4.58 \pm 0.20^{h-t}$ & $5.10 \pm 0.10^{-y y}$ & $67.21 \pm 0.01^{n-t}$ & $71.45 \pm 0.02^{\mathrm{a}-0}$ & $72.40 \pm 0.03^{y-m}$ \\
\hline & & 30 & $4.43 \pm 0.06^{k-u}$ & $4.54 \pm 0.20^{i-u}$ & $5.00 \pm 0.11^{t-y}$ & $66.07 \pm 1.76^{p-y}$ & $68.23 \pm 0.01^{\mathrm{m}-\mathrm{r}}$ & $70.29 \pm 0.64^{a-0}$ \\
\hline & & 35 & $4.36 \pm 0.04^{a-h}$ & $4.45 \pm 0.10^{k-u}$ & $4.65 \pm 0.04^{f-5}$ & $64.58 \pm 0.17^{-b}$ & $66.78 \pm 0.01^{0-x}$ & $67.96 \pm 0.03^{\mathrm{m}-1}$ \\
\hline & & 40 & $4.28 \pm 0.02^{a-5}$ & $4.42 \pm 0.08^{k-u}$ & $4.32 \pm 0.01^{\mathrm{ath}}$ & $63.26 \pm 0.02^{r-e}$ & $65.07 \pm 0.36^{r-b}$ & $65.77 \pm 0.01^{p-z}$ \\
\hline & \multirow{9}{*}{ 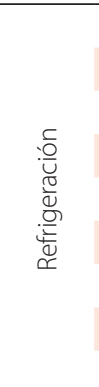 } & 1 & $7.23 \pm 0.08^{\text {cd }}$ & $9.02 \pm 0.66^{y-a}$ & $8.29 \pm 1.47^{2-c y}$ & $72.93 \pm 0.45^{y-m}$ & $83.38 \pm 0.28^{a}$ & $82.46 \pm 0.21^{f_{-0}}$ \\
\hline & & 5 & $6.00 \pm 0.18^{\mathrm{ef}}$ & $7.03 \pm 0.21^{\mathrm{cd}}$ & $7.00 \pm 0.74^{\mathrm{de}}$ & $73.80 \pm 0.02^{w-y}$ & $82.32 \pm 0.03^{\mathrm{ab}}$ & $81.66 \pm 0.02^{9-0}$ \\
\hline & & 10 & $4.63 \pm 0.33^{g-t}$ & $4.97 \pm 0.12^{b-5}$ & $5.63 \pm 0.09^{f}$ & $73.73 \pm 0.68^{x-k}$ & $81.11 \pm 0.86 \mathrm{~g}-0$ & $80.82 \pm 0.22^{1-0}$ \\
\hline & & 15 & $4.56 \pm 0.17^{h-t}$ & $4.67 \pm 0.06^{\mathrm{e}-5}$ & $5.30 \pm 0.05^{p-y}$ & $72.93 \pm 0.02^{y-m}$ & $79.71 \pm 0.01^{1-0}$ & $79.42 \pm 0.02^{j-0}$ \\
\hline & & 20 & $4.48 \pm 0.12^{j-u}$ & $4.40 \pm 0.08^{m-w}$ & $4.92 \pm 0.03^{b-5}$ & $72.52 \pm 0.19^{y-m}$ & $78.55 \pm 0.24^{-0}$ & $78.25 \pm 0.19^{k-0}$ \\
\hline & & 25 & $4.39 \pm 0.07^{\text {a-h }}$ & $4.39 \pm 0.04^{\mathrm{a}-\mathrm{h}}$ & $4.81 \pm 0.02^{c-5}$ & $72.07 \pm 0.02^{2-n}$ & $77.10 \pm 0.01^{1-0}$ & $77.17 \pm 0.01^{k-0}$ \\
\hline & & 30 & $4.31 \pm 0.01^{a-1}$ & $4.38 \pm 0.01^{a-h}$ & $4.71 \pm 0.02^{\mathrm{d}-5}$ & $71.39 \pm 0.89^{t-1}$ & $75.19 \pm 0.53^{n 0}$ & $75.69 \pm 0.90^{\text {no }}$ \\
\hline & & 35 & $4.27 \pm 0.01^{1-5}$ & $4.31 \pm 0.04^{\mathrm{a}-\mathrm{h}}$ & $4.40 \pm 0.18^{m-x}$ & $71.21 \pm 0.02^{t-1}$ & $74.49 \pm 0.02^{\circ}$ & $74.93 \pm 0.02^{\text {no }}$ \\
\hline & & 40 & $4.24 \pm 0.01^{-1-k}$ & $4.27 \pm 0.05^{\mathrm{a}-\mathrm{s}}$ & $4.28 \pm 0.02^{2-5}$ & $70.86 \pm 0.18^{t-i}$ & $73.54 \pm 0.29^{y-m}$ & $74.04 \pm 0.29^{\circ}$ \\
\hline
\end{tabular}




\section{Ácido ascórbico e intensidad respiratoria (IR)}

Otros parámetros que disminuyeron significativamente con el tiempo de almacenamiento fueron el contenido de ácido ascórbico y la intensidad respiratoria $(p<0.05)$. Tukey al $5 \%$ para la interacción "variedades $x$ tratamiento $x$ condición de almacenamiento $x$ tiempo de almacenamiento" identificó 25 rangos de significación. En el primer rango con el mayor contenido de ácido ascórbico se encuentra la variedad chaucha no irradiada, almacenada al ambiente y en refrigeración por un día con 9.34 mg/100 g y en el último rango de significación con el menor contenido de ácido ascórbico se ubica la variedad chola no irradiada, almacenada en refrigeración por 40 días, con 3.36 mg/100 g. El ácido ascórbico o vitamina $C$ es un agente antioxidante necesario para la formación y mantenimiento adecuado del material intercelular; puede reducir la acción perjudicial de los radicales libres y coadyuva al mejoramiento de la absorción del hierro no hemínico [21, 22]. Es muy sensible a la luz, temperatura y oxígeno degradándose fácilmente durante el procesamiento y almacenamiento de los alimentos [21]. En el monitoreo del tiempo de almacenamiento se determinó que el ácido ascórbico disminuyó súbitamente en los primeros 10 días de almacenamiento, en un $45 \%$ en la papa irradiada y $62.36 \%$ en la papa sin tratamiento de irradiación. Lo que evidencia la sensibilidad de este nutriente al almacenamiento, la radiación y el oxígeno del aire, a través de las oxidasas catalíticas y/o trazas de algunos metales. Según varios autores, la oxidasa del ácido ascórbico está ampliamente distribuida en los tejidos de las plantas y cataliza la reacción hasta ácido dehidroascórbico y una reacción estrechamente relacionada tiene lugar para la reacción directa del oxígeno del aire, mostrando que la radiación no inactiva las enzimas de la papa $[15,18]$. Al término del periodo de almacenamiento estas pérdidas alcanzaron el $54 \%$ en la papa irradiada y 65\% en la papa no irradiada (Tabla 2), evidenciando el efecto de deterioro en almacenamiento. Estos resultados concuerdan con los reportados por otros autores, quienes registraron pérdidas de ácido ascórbico en el orden del $64 \%$ en papas almacenadas por dos meses a $18^{\circ} \mathrm{C}[3,15]$.

La respiración es un proceso vital que proporciona la energía requerida para mantener vivos los tejidos de los tubérculos. En este proceso, los azúcares son convertidos en agua y dióxido de carbono mediante la captación de oxígeno del ambiente, liberando a su vez calor [20]. La prueba de Tukey al 5\% para la interacción "variedades $x$ tratamiento $x$ condición de almacenamiento $x$ tiempo de almacenamiento" identificó 40 rangos de significación. En el primer rango con la mayor intensidad respiratoria se encuentra la variedad chaucha irradiada, almacenada al ambiente por un día con $83.38 \mathrm{mgCO} / \mathrm{kg}^{*}$ hy en el último rango de significación con la menor intensidad respiratoria se ubica la variedad chaucha no irradiada, almacenada en condición de ambiente por 40 días, con $1.07 \mathrm{mgCO} / \mathrm{kg}^{*} \mathrm{~h}$. Durante la respiración la producción de energía proviene de la oxidación de las propias reservas de almidón, azucares y otros metabolitos. Una vez cosechada, la papa no puede reemplazar estas reservas que se pierden y la velocidad con que disminuyen es un factor de gran importancia en la duración de la vida poscosecha del tubérculo [12]. La intensidad respiratoria (IR) de la papa, disminuyó con el tiempo de almacenamiento, en mayor proporción y velocidad en los tubérculos no irradiados y almacenados en condiciones de ambiente (Tabla 2); en las variedades chaucha y ratona no irradiadas y almacenadas al ambiente, la IR disminuyó en un 94\%, mientras que en la variedad chola almacenada a $7^{\circ} \mathrm{C}$, la IR disminuyó en un $21.43 \%$ con respecto a la papa recién cosechada. La irradiación de los tubérculos y una menor temperatura de almacenamiento atenuaron la disminución de la IR a un promedio de 13\%, en las tres variedades en estudio; bajo estas condiciones la IR de la variedad ratona en refrigeración, sólo disminuyó un 6.25\%, 
con respecto al valor inicial $\left(80 \mathrm{mgCO} / \mathrm{kg}^{*} \mathrm{~h}\right)$. Estos resultados concuerdan con los reportados por Rezaee et al. (2011) y Prakash et al. (2000) [3, 4], quienes indican que las bajas temperaturas de almacenamiento ayudan a disminuir la velocidad de respiración y a prolongar la durabilidad postcosecha de la papa.

\section{Color del tegumento y tamaño de los brotes}

A diferencia de los componentes nutricionales e intensidad respiratoria, la diferencia de color total $(\Delta \mathrm{E})$ del tegumento y el tamaño de los brotes aumentaron con el tiempo de almacenamiento $(p<0.05)$ (Tabla 3). La prueba de Tukey al $5 \%$ para la interacción "variedades $\mathrm{x}$ tratamiento $\mathrm{x}$ condición de almacenamiento $\mathrm{x}$ tiempo de almacenamiento" identificó 35 rangos de significación. En el primer rango con la menor diferencia de color se encuentra la variedad ratona irradiada, almacenada al ambiente por cinco días con un $(\triangle \mathrm{E})$ de 185.07 y en el último rango de significación con el mayor $\Delta \mathrm{E}$ (382.39) se ubica la variedad chola no irradiada, almacenada en refrigeración entre 35-40 días. En general, El color es un atributo de apariencia de la papa, depende de varios factores y tiene importancia desde el punto de vista comercial ya que determina su grado de aceptación por el consumidor. Los tubérculos almacenados en refrigeración, sin el tratamiento de irradiación, experimentaron una mayor variación $(\Delta \mathrm{E})$, con respecto al color inicial, especialmente las variedades chaucha y ratona. Los resultados muestran que la refrigeración provocó un mayor efecto que la irradiación sobre el color del tegumento, ya que los tubérculos que fueron irradiados y almacenados al ambiente experimentaron menor variación de color. Este resultado también sugiere que el límite superior de dosis para la irradiación de la papa corresponde a $120 \mathrm{~Gy}$, dosis mayores pueden provocar un oscurecimiento de la papa [12].

En cuanto al tamaño de los brotes, desde el inicio de la formación de los tubérculos hasta el momento de la cosechay parte inicial del período de almacenamiento las papas se encuentran en estado de "dormancia"; es decir, no brotan por estar bajo el efecto de inhibidores naturales. Este período duró entre a 3 a 5 días en las variedades chaucha y ratona, no irradiadas y almacenadas a $12^{\circ} \mathrm{C}$ y $70 \%$ de humedad relativa, mientras que el periodo de dormancia de la variedad chola, superó los 40 días de monitoreo, sin demostración visible del crecimiento de brotes (Tabla 3), resultado que puede tener repercusiones económicas, al prolongar la vida útil de las papas nativas disminuyendo las pérdidas postcosecha [24]. La variedad chaucha, almacenada a $12^{\circ} \mathrm{C}$, sin irradiar, presentó dormancia de 10 a 15 días a $7^{\circ} \mathrm{C}$. El período de dormancia de la variedad ratona sin irradiar fue de 20 días a $12^{\circ} \mathrm{C}$ y 25 días a $7^{\circ} \mathrm{C}$. La prueba de Tukey al $5 \%$ para la interacción "variedades $x$ tratamiento x condición de almacenamiento $x$ tiempo de almacenamiento" identificó 12 rangos de significación. En el primer rango con ausencia de brotes se ubicaron las tres variedades, irradiadas, almacenadas al ambiente y en refrigeración de 5 a 40 días y en el último rango de significación con el mayor crecimiento de brotes se ubicó la variedad ratona no irradiada, almacenada al ambiente por 40 días. Generalmente se considera que la dormancia se pierde cuando un tubérculo contiene uno o más brotes con una longitud mayor de dos milímetros [20]. Con el tratamiento de irradiación, independientemente de la temperatura de almacenamiento, el período de dormancia de las tres variedades de papa superó los 40 días de monitoreo y prueba, lo cual según Viola et al. [20] podría relacionarse con la presencia de aislamiento simplástico en el brote apical, que retarda la conversión de carbohidratos almacenados en azúcares disponibles para el inicio de la brotación. Siendo éste resultado uno de los mayores efectos perceptibles en la papa irradiada. 
Tabla 3. Variación del color y tamaño de los brotes por efecto del tratamiento, condición y tiempo de almacenamiento de tres variedades de papa

\begin{tabular}{|c|c|c|c|c|c|c|c|c|}
\hline \multirow[b]{2}{*}{$\begin{array}{l}\text { Trata- } \\
\text { miento }\end{array}$} & \multirow[b]{2}{*}{$\begin{array}{c}\text { Almace- } \\
\text { namiento }\end{array}$} & \multicolumn{4}{|c|}{ Diferencia total de color del tegumento $(\Delta \mathrm{E})$} & \multicolumn{3}{|c|}{ Tamaño de los brotes (mm) } \\
\hline & & Días & Chola & Chaucha & Ratona & Chola & Chaucha & Ratona \\
\hline \multirow{16}{*}{ 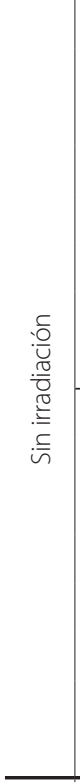 } & \multirow{8}{*}{ 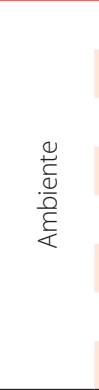 } & 5 & $249.50 \pm 1.39^{d-p}$ & $215.64 \pm 0.58^{\text {ae }}$ & $229.90 \pm 0.38^{i-u}$ & $0.00^{2}$ & $1.00 \pm 0.01^{b c d}$ & $1.00 \pm 0.01^{\text {bd }}$ \\
\hline & & 10 & $255.29 \pm 1.96^{d-p}$ & $221.17 \pm 0.58^{\text {af }}$ & $253.35 \pm 0.38^{d-p}$ & $0.00^{3}$ & $1.67 \pm 0.01^{\text {def }}$ & $1.00 \pm 0.01^{\text {bd }}$ \\
\hline & & 15 & $259.02 \pm 1.33^{e-r}$ & $229.98 \pm 0.58^{-u}$ & $277.56 \pm 0.38^{k-x}$ & $0.00^{\mathrm{a}}$ & $3.00 \pm 0.02^{\text {hij }}$ & $1.00 \pm 0.01^{\text {bcd }}$ \\
\hline & & 20 & $267.98 \pm 1.48^{9-t}$ & $238.48 \pm 0.55^{b-k}$ & $288.73 \pm 0.38^{M-b}$ & $0.00^{3}$ & $3.67 \pm 0.03^{\mathrm{k}}$ & $1.33 \pm 0.01^{\text {def }}$ \\
\hline & & 25 & $273.70 \pm 0.777^{-w}$ & $245.99 \pm 0.58^{d-n}$ & $312.56 \pm 0.38^{\text {Tf }}$ & $0.00^{3}$ & $5.00 \pm 0.04^{L}$ & $2.67 \pm 0.02^{k}$ \\
\hline & & 30 & $280.27 \pm 1.95^{k-z}$ & $253.38 \pm 0.28^{d-p}$ & $332.63 \pm 0.49^{-h}$ & $0.00^{3}$ & $5.00 \pm 0.04$ & $3.67 \pm 0.02$ \\
\hline & & 35 & $286.99 \pm 1.39^{-a}$ & $261.11 \pm 0.28^{e-r}$ & $352.70 \pm 0.49^{f-j}$ & $0.00^{\mathrm{a}}$ & $5.00 \pm 0.03^{L}$ & $4.67 \pm 0.03^{L}$ \\
\hline & & 40 & $293.61 \pm 0.78^{0-c}$ & $268.84 \pm 0.28^{9-u}$ & $372.77 \pm 0.49^{h-j}$ & $0.00^{2}$ & $5.00 \pm 0.04^{L}$ & $5.00 \pm 0.03^{L}$ \\
\hline & \multirow{8}{*}{ 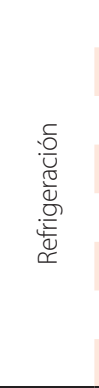 } & 5 & $246.66 \pm 0.64^{d-n}$ & $192.83 \pm 0.28^{\mathrm{abb}}$ & $271.30 \pm 0.49^{9-\mathrm{t}}$ & $0.00^{2}$ & $1.00 \pm 0.01^{b c d}$ & $1.00 \pm 0.01^{\mathrm{bcd}}$ \\
\hline & & 10 & $268.69 \pm 2.26^{9-t}$ & $208.95 \pm 0.28^{2-d}$ & $292.62 \pm 0.49^{W-c}$ & $0.00^{3}$ & $1.67 \pm 0.01^{\text {def }}$ & $1.00 \pm 0.01^{\text {bd }}$ \\
\hline & & 15 & $277.93 \pm 1.35^{k y}$ & $236.81 \pm 0.28^{b-k}$ & $305.27 \pm 0.49^{\text {Re }}$ & $0.00^{\mathrm{a}}$ & $2.33 \pm 0.02^{\text {tgh }}$ & $1.00 \pm 0.01^{\mathrm{bcd}}$ \\
\hline & & 20 & $288.95 \pm 0.60^{\mathrm{M}-\mathrm{c}}$ & $268.05 \pm 0.28^{g-t}$ & $318.52 \pm 0.49^{W-f}$ & $0.00^{3}$ & $3.33 \pm 0.02^{i j}$ & $1.67 \pm 0.01^{\text {def }}$ \\
\hline & & 25 & $304.51 \pm 2.18^{0-e}$ & $290.04 \pm 0.47^{\mathrm{N}-\mathrm{C}}$ & $335.51 \pm 0.499^{-i}$ & $0.00^{\mathrm{a}}$ & $4.33 \pm 0.03^{W}$ & $2.00 \pm 0.03^{\text {feq }}$ \\
\hline & & 30 & $318.53 \pm 1,07^{W-f}$ & $315.39 \pm 0.47^{U-f}$ & $350.94 \pm 0.49^{-j}$ & $0.00^{3}$ & $4.67 \pm 0.04^{L}$ & $2.33 \pm 0.02^{\text {gh }}$ \\
\hline & & 35 & $382.39 \pm 0.49 j$ & $340.74 \pm 0.47^{d-j}$ & $366.37 \pm 0.49^{9-j}$ & $0.00^{3}$ & $5.00 \pm 0.04$ & $3.33 \pm 0.02$ \\
\hline & & 40 & $346.25 \pm 0.56 \mathrm{e}-\mathrm{j}$ & $366.1 \pm 0.47^{9-j}$ & $381.81 \pm 0.49 j$ & $0.00^{3}$ & $5.00 \pm 0.04^{L}$ & $4.33 \pm 0.03^{\mathrm{k}}$ \\
\hline \multirow{16}{*}{ 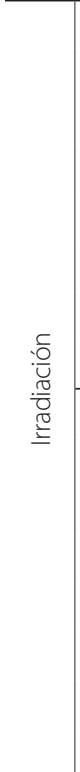 } & \multirow{8}{*}{ 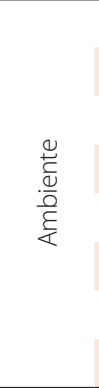 } & 5 & $233.70 \pm 2.24^{b-k}$ & $216.29 \pm 0.47^{\text {ae }}$ & $185.06 \pm 0.50^{\mathrm{a}}$ & $0.00^{\mathrm{a}}$ & $0.00 \pm 0.00^{\mathrm{a}}$ & $0.00^{\mathrm{a}}$ \\
\hline & & 10 & $235.04 \pm 0.38^{b-k}$ & $217.87 \pm 0.47^{\text {ae }}$ & $197.25 \pm 0.50^{\mathrm{acc}}$ & $0.00^{2}$ & $0.00 \pm 0.00^{3}$ & $0.00^{3}$ \\
\hline & & 15 & $235.85 \pm 1.41^{b-k}$ & $218.72 \pm 0.47^{-\mathrm{af}}$ & $227.07 \pm 0.42^{2-i}$ & $0.00 \mathrm{a}$ & $0.00 \pm 0.00^{\mathrm{a}}$ & $0.00^{2}$ \\
\hline & & 20 & $236.44 \pm 1.42^{b-k}$ & $219.39 \pm 0.40^{2 . f}$ & $258.85 \pm 0.42^{e-r}$ & $0.00 \mathrm{a}$ & $0.00 \pm 0.00^{\mathrm{a}}$ & $0.00^{3}$ \\
\hline & & 25 & $237.80 \pm 2.04^{b-k}$ & $219.39 \pm 0.40^{3-f}$ & $269.08 \pm 0.42^{h-u}$ & $0.00 \mathrm{a}$ & $0.00 \pm 0.00^{\mathrm{3}}$ & $0.00^{\mathrm{a}}$ \\
\hline & & 30 & $239.23 \pm 2.00^{b-k}$ & $219.39 \pm 0.42^{2-9}$ & $294.99 \pm 0.40^{p-d}$ & $0.00 \mathrm{a}$ & $0.00 \pm 0.00^{\mathrm{a}}$ & $0.00^{3}$ \\
\hline & & 35 & $240.15 \pm 1.50^{c k}$ & $219.39 \pm 0.42^{\text {ath }}$ & $311.09 \pm 0.40^{s-f}$ & $0.00 \mathrm{a}$ & $0.00 \pm 0.00^{\mathrm{a}}$ & $0.00^{3}$ \\
\hline & & 40 & $241.28 \pm 2.31^{-1}$ & $219.39 \pm 0.42^{2-i}$ & $325.24 \pm 0.40^{2-9}$ & $0.00^{2}$ & $0.00 \pm 0.00^{\mathrm{B}}$ & $0.00^{3}$ \\
\hline & \multirow{8}{*}{ 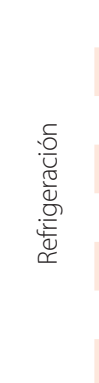 } & 5 & $225.17 \pm 0.59^{2-i}$ & $216.29 \pm 0.43^{\text {ath }}$ & $322.15 \pm 0.40^{x-9}$ & $0.00^{3}$ & $0.00 \pm 0.00^{\mathrm{a}}$ & $0.00^{3}$ \\
\hline & & 10 & $230.85 \pm 0.8 g^{b-k}$ & $216.99 \pm 0.43^{2 \mathrm{~h}}$ & $324.27 \pm 0.40^{\gamma-9}$ & $0.00^{3}$ & $0.00 \pm 0.00^{\mathrm{a}}$ & $0.00^{3}$ \\
\hline & & 15 & $236.08 \pm 1.94^{b-k}$ & $219.39 \pm 0.37^{\text {th }}$ & $325.79 \pm 0.40^{2-9}$ & $0.00^{2}$ & $0.00 \pm 0.00^{\mathrm{a}}$ & $0.00^{\circ}$ \\
\hline & & 20 & $242.34 \pm 2,200^{-m}$ & $219.39 \pm 0.37^{-\mathrm{th}}$ & $326.96 \pm 0.38^{a}-h$ & $0.00^{3}$ & $0.00 \pm 0.00^{\mathrm{a}}$ & $0.00^{3}$ \\
\hline & & 25 & $247.00 \pm 0.55^{d-0}$ & $219.39 \pm 0.37^{-i-1}$ & $329.43 \pm 0.38^{\text {th }}$ & $0.00^{\mathrm{a}}$ & $0.00 \pm 0.04^{a}$ & $0.00^{\mathrm{a}}$ \\
\hline & & 30 & $250.21 \pm 0.40^{d-p}$ & $219.39 \pm 0.37^{-i-1}$ & $331.62 \pm 0.38^{\text {th }}$ & $0.00^{3}$ & $0.00 \pm 0.04^{a}$ & $0.00^{\mathrm{a}}$ \\
\hline & & 35 & $257.91 \pm 1.80^{e-q}$ & $219.39 \pm 0.37^{-i-1}$ & $333.06 \pm 0.38^{-\mathrm{ah}}$ & $0.00^{3}$ & $0.00 \pm 0.04^{\mathrm{a}}$ & $0.00^{3}$ \\
\hline & & 40 & $264.60 \pm 0.47^{\text {t.s }}$ & $219.39 \pm 0.30^{2-1}$ & $334.84 \pm 0.38^{\text {b-h }}$ & $0.00^{3}$ & $1.00 \pm 0.04^{\text {bed }}$ & $0.00^{3}$ \\
\hline
\end{tabular}




\section{Durabilidad}

La representación gráfica de la variación de humedad con el tiempo de almacenamiento, correspondió a una cinética de orden cero ( $p$ < 0.05) (Tabla 4) y se observó un buen ajuste con los datos experimentales, con coeficientes de correlación entre 0.97 y 1.00. A partir de las ecuaciones descritas en la Tabla 4 se realizó la predicción del tiempo en que la papa alcanzaría el 65 \% de humedad, considerado como límite crítico de aceptabilidad para el consumo en estado fresco, bajo este nivel los tubérculos presentaron arrugamiento, pérdida de coloración y crecimiento de brotes. Se determinó una menor durabilidad para los tubérculos almacenados al ambiente y sin tratamiento de irradiación, en el siguiente orden: 17 días (ratona), 28 días (chaucha) y 48 días (chola). El almacenamiento en refrigeración permitiría aumentar la durabilidad de las tres variedades a 19 días (ratona), 45 días (chaucha y 69 días (chola). Cuando los tubérculos se irradian, la durabilidad en condición de ambiente se prolongaría a 38 días (ratona), 58 días (chaucha), 80 días (chola). El efecto sinérgico de la irradiación y la refrigeración prolongaría la durabilidad de los tubérculos a 44 días (ratona), 114 días (chaucha y 106 días (chola), retardando su descomposición y reduciendo su infestación por insectos y/o la contaminación por otros organismos.

Tabla 4. Cinética de la variación de humedad de papa con el tiempo de almacenamiento

\begin{tabular}{|c|c|c|c|c|}
\hline $\begin{array}{l}\text { Trata- } \\
\text { miento }\end{array}$ & $\begin{array}{l}\text { Almace- } \\
\text { namiento }\end{array}$ & Chola & Chaucha & Ratona \\
\hline \multirow{2}{*}{ 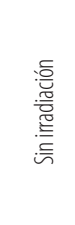 } & 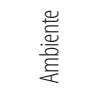 & $y=-0.375 x+77.88\left(r^{2}=0.9877\right)$ & $y=-0.609 x+81.87\left(r^{2}=0.9912\right)$ & $y=-1.109 x+83.39\left(r^{2}=0.9838\right)$ \\
\hline & 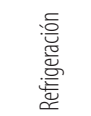 & $y=-0.257 x+77.66\left(r^{2}=0.9950\right)$ & $y=-0.382 x+82.20\left(r^{2}=0.9869\right)$ & $y=-0.968 x+83.13\left(r^{2}=0.97795\right)$ \\
\hline \multirow{2}{*}{ 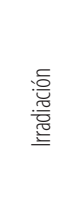 } & 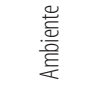 & $y=-0.251 x+80.07\left(r^{2}=0.9866\right)$ & $y=-0.287 x+81.57\left(r^{2}=0.9744\right)$ & $y=-0.477 x+83.09\left(r^{2}=0.9911\right)$ \\
\hline & 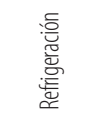 & $y=-0.188 x+80.02\left(r^{2}=0.9698\right)$ & $y=-0.145 x+81.54\left(r^{2}=0.9894\right)$ & $y=-0.411 x+83.15\left(r^{2}=0.9750\right)$ \\
\hline
\end{tabular}

\section{CONCLUSIONES}

Los mayores cambios en los contenidos de almidón y los azúcares se produjeron en los tubérculos no irradiados y almacenados en condiciones de ambiente $\left(12^{\circ} \mathrm{C}\right.$ y $70 \%$ de humedad relativa).

La radiación gamma retardó los cambios bioquímicos y la intensidad respiratoria de la papa, efecto que fue potencializado con la baja temperatura de almacenamiento permitiendo extender la vida útil de la variedad ratona hasta 44 días y de chaucha hasta 114 días. No obstante, la irradiación de alimentos a mayor escala está sujeta a la 
disponibilidad de una fuente industrial de cobalto $60\left(\mathrm{CO}_{60}\right)$, que se encuentra hoy en día bajo consideración y estudio del Ministerio de Producción, comercio Exterior, Inversiones y Pesca, MPCEIP.

El control de algunos factores que controlan la brotación acelerada mediante la irradiación es de gran importancia para fitomejoradores, productores y procesadores de papa en estado fresco, en la medida en que constituyen herramientas para mejorar la competitividad y generar nuevas opciones para el desarrollo agrícola de Ecuador.

Este estudio presenta información que puede ayudar a los diferentes actores a decidir con sensatez sobre el lugar que debe ocupar la irradiación en sus esfuerzos por garantizar un suministro de papa suficiente, sano y fiable.

\section{AGRADECIMIENTOS}

A la Agencia Internacional de Energía Atómica, por el apoyo financiero para realizar la presente investigación. Al Instituto Nacional de Investigaciones Agropecuarias, por el apoyo y las facilidades brindadas para realizar el estudio. Un resumen del presente trabajo se presentó en el II Simposio Latinoamericano de Aplicaciones Nucleares en la Agricultura.

\section{CONTRIBUCIÓN DE LOS AUTORES}

Elena Villacrés; mentalizó la investigación, diseñó los experimentos, redactó el manuscrito y participó como tutor y revisor de los resultados. Mishel Yánez realizó los ensayos de laboratorio, la toma de datos y análisis estadístico de resultados. María Belén Quelal participó en la validación de las metodologías aplicadas. Trosky Yánez contribuyó en la revisión de los resultados de la investigación. 


\section{REFERENCIAS}

[1] Devaux, A., Kromann, P., Ortiz, 0. (2014) Potatoes for sustainable global food security. Potato Res 57:185-199

[2] Basantes, F., Suárez, J.P.A., Illescas, L.M.A., Hernández, L. del R.V. (2020). Diagnóstico de la situación actual de la producción y comercialización de la papa (Solanum tuberosum L.) en la Zona 1 del Ecuador. E-Agronegocios 6:103-120

[3] Rezaee, M., Almasi, M., Majdabadi Farahani, A., Minaei, S., \& Khodadadi, M. (2011). Potato sprout inhibition and tuber quality after post harvest treatment with gamma irradiation on different dates. Journal of Agricultural Science and Technology, 13(6), 829-842

[4] Prakash, A., Guner, A.R., Caporaso, F., Foley, D.M. (2000). Effects of low dose gamma irradiation on the shelf life and quality characteristics of cut romaine lettuce packaged under modified atmosphere. J Food Sci 65:549-553

[5] Mejía Villafuerte, D.A. (2017). Pérdidas poscosecha en la cadena de valor del rubro papa, un estudio de caso en la provincia del Carchi Año: 2016. (Bachelor's thesis, PUCE). Quito, Ecuador.

[6] Rodriguez, L.E, Moreno, L.P. (2010). Factores y mecanismos relacionados con la dormancia en tubérculos de papa. Una revisión. Agron Colomb 38:189-197

[7] Daniels Lake, B., Olsen, N., López Delgado, H., Zink, R. (2013). Eficacia de los productos controladores de la brotación de papa. Doc Ciency Tecnol la NAPPO 3-4.

[8] Arvanitoyannis, I.S, Stratakos, A.C, Tsarouhas, P. (2009). Irradiation applications in vegetables and fruits: a review. Crit Rev Food Sci Nutr 49:427-462

[9] Singh, R. P., \& Heldman, D. R. C. (2009). Introducción a la ingeniería de los alimentos.No. 641.3 Si646i . Editorial Acribia, Zaragoza, España. 200p.

[10] Afshari, H. (2006). Examination of Mechanical Properties and Impact Sensitivity of Potato Varieties and Mathematical Modeling of Tuber Damage Susceptibility (Doctoral dissertation, Ph. D. Thesis. Mechanics of Farm Machinery, Faculty of Agriculture, Sciences and Researches Branch, Islamic Azad University. Iran).

[11] Suárez, R. (2001). Conservación de alimentos por irradiación. Invenio 4:85-124

[12] Ruiz Ramos, E. T. (2016). Estudio del efecto del tratamiento poscosecha por irradiación gamma sobre la vida útil de la cebolla perla ecuatoriana de exportación (Allium cepa L.) (Bachelor's thesis). Escuela Politéncia Nacional, Quito.

[13] AOAC (Association of Official Analytical Chemestry) № Title. In: Horwitz W (ed) 17 th. Arlington

[14] Durán, L., Fiszman, S., Benedito, C. (2001). Propiedades mecánicas empíricas: Métodos para medir propiedades físicas industriales de alimentos. Métodos para medir propiedades físicas en Ind Aliment Prim edición Ed Acribia, SA, Zaragoza, España 153-154

[15] Egoville, M.J, Sullivan, J.F, Kozempel, M.F., Jones, W.J. (1988). Ascorbic acid determination in processed potatoes. Am Potato J 65:91-97

[16] Buitrago, G. V., López, A.P., Coronado, A.P., Osorno, F.L. (2004). Determinación de las características físicas y propiedades mecánicas de papa cultivada en Colombia. Rev Bras Eng Agrícola e Ambient 8:102-110

[17] Gallo Pérez, F. (1996). Manual de fisiología, patología post-cosecha y control de calidad de frutas y hortalizas. SENA-NRI, Colombia. 180p.

[18] Alvarado, J. de D. (1996). Principios de ingeniería aplicados a alimentos. División de artes gráficas. en una licuadora Oster por $3 \mathrm{~min}, 19$. Claassens MMJ (2002) Carbohydrate metabolism during potato tuber dormancy and sprouting

[19] Viola, R., Pelloux, J., van der Ploeg, A., et al. (2007). Symplastic connection is required for bud outgrowth following dormancy in potato (Solanum tuberosum L.) tubers. Plant Cell Environ 30:973-983

[20] Grosso, G., Bei, R., Mistretta, A., Marventano, S., Calabrese, G., Masuelli, L., Gazzolo, D. (2013). Effects of vitamin Con health: A review of evidence, Front Biosci-Landmark 18:1017-29. 
[21] Shaik-Dasthagirisaheb, Y.B., Varvara, G., Murmura, G., Saggini, A., Caraffa, A., Antinolfi, P., Pandolfi, F. (2013). Role of vitamins D, E and C in immunity and inflammation. J Biol Reg Homeos Ag. 27(2):291-5.

[22] Organización Mundial de la Salud, (1989). La irradiación de los alimentos. Gráficas Reunidas, Zaragoza, España. 90p.

[23] Velástegui-Espín, G. P., Artieda-Rojas, J. R., Mera-Andrade, R. I., López-Villacís, I. C., Pazmiño-Miranda, N. D. P., \& Espinoza-Vaca, J. S. (2018). Inhibición de la brotación del tubérculo de papa: una revisión de los métodos empleados. Journal of the Selva Andina Biosphere, 6(2), 55-64. 